\title{
The effect of the thrombolytic therapy on the early rehabilitation of patients with acute ischemic stroke - study report
}

\section{MUTU Cătălin Cosmin ${ }^{1,2}$, CEREI Larisa-Georgiana ${ }^{1}$}

Corresponding author: MUTU Cătălin Cosmin, E-mail: $\underline{\text { cosmin.mutu@ulbsibiu.ro }}$

1. "Lucian Blaga" University of Sibiu, Romania

2. Clinical County Emergency Hospital, CVASIC Research Center, Sibiu, Romania

\begin{abstract}
Introduction. r-TPA intravenous thrombolytic therapy is a big step in acute management of ischemic stroke (IS) but is burdened by a small therapeutic window (max. 4.5 hours) that restricts patient access. NIH stroke scale (NIHSS) is the largest worldwide tool used by doctors in order to objectively quantify the severity of neurological impairment in acute IS, in the initial stage as well as in the subsequent stages. Material and method. The aim of this paper is to present the results of an observational, prospective, analytical study on a number of 110 patients with acute IS, hospitalized within two months in the Neurology Clinic of Sibiu (19 patients with thrombolytic therapy applied, 91 patients with classic therapy). NIHSS has been applied to all patients on the first day of the admission and at the discharge date. Results and discussions. The mean value of NIHSS at admission and at discharge (A/D) were 12,84/4.06 points for the patient with thrombolytic therapy and 7,73/4,45 points for the patients without thrombolytic therapy. In hospital NIHSS score reduction, meaning early recovery of patients, was consistent with type of applied therapy $(8.78$ vs3.28 points). The pattern of neurological impairment is specific for each subtype of IS. Conclusions. The degree of early recovery was superior for patients with versus without thrombolytic therapy $(68.4 \%$ versus $42.4 \%)$. Average hospital care period was reduced with $8,33 \%$ for patients with thrombolytic therapy (11,4 versus 12,2 days care in hospital).
\end{abstract}

Keywords: ischemic stroke, early rehabilitation, NIH stroke scale, neurological pattern,

\section{Introduction}

Stroke represents one of the leading causes of morbidity, mortality and persistent disability worldwide. Ischemic stroke is a heterogeneous disease with distinct subtypes, each of them presenting specific etiological, pathogenesis and clinical aspects (1).

The widespread introduction of IV thrombolytic therapy with r-TPA in Romania, starting with January 2019, has probably significantly improved the chances of patients with acute ischemic stroke for a favorable evolution. Unfortunately, patients access to thrombolytic therapy is limited by the reduced window of time to only 4.5 hours after the onset of a stroke.

As clinician-reported scales of global disability, modified Rankin Scale (mRS) and National Institute of Health Stroke Scale (NIHSS) are the tools used by doctors in order to objectively quantify the severity of neurological impairment in acute ischemic stroke, in the initial stage as well as in the subsequent stages $(1,2,3)$.

Patient-reported scales to be used in the primary care medicine are: The Patient-Reported Outcome Measure Information System (PROMIS) and Quality of Life in Neurological Disorders (NeuroQoL) scales $(4,5)$.

\section{Purpose}

The aim of this study was to evaluate the neurological impairment, as well as the assessment of the short-term evolution of inpatients in the Neurology Clinic of Sibiu, by analyzing the dynamics of all 11 items of the NIHSS in the two key moments of hospitalization (admission and discharge) taking into consideration the access / lack of access of these patients to thrombolytic therapy.

\section{Materials and methods}

We conducted an observational, prospective, analytical study of 110 patients with acute ischemic stroke, hospitalized within two months in the Neurology Clinic of Sibiu (March-April 2019). The patients were divided into two groups according to the therapy applied (patients with or without thrombolysis). NIHSS has been evaluated in all patients on the first day of the admission and at the discharge date. The primary objective was to establish correlations between the recovery degree of patients and the type of applied therapy. The secondary objective was to determine the pattern of neurological damage in patients included in study and to achieve correlations between the extent of neurological damage, type of therapy applied and the degree of patients' early recovery.

\section{Results}

All 110 patients with acute ischemic stroke (mean age $71,42+/-20$ years) hospitalized during the study period were included.

In respect to the type of therapy, the patients were divided into two subgroups with specific characteristics presented in the synoptic table below. 
Table 1. Patients' demographic and clinical characteristics according to applied therapy

\begin{tabular}{|l|l|l|l|}
\hline Items & Total & $\begin{array}{l}\text { r-TPA } \\
\text { treated }\end{array}$ & $\begin{array}{l}\text { Non r- } \\
\text { TPA } \\
\text { treated }\end{array}$ \\
\hline Patients & $110(100)$ & $19(17.3)$ & $91(82.7)$ \\
\hline Mean age: $y$ & 71.9 & 71.94 & 71.91 \\
\hline Female & $50(45.4)$ & $8(42.1)$ & $42(46.1)$ \\
\hline Male & $60(54.5)$ & $11(57.9)$ & $49(53.8)$ \\
\hline $\begin{array}{l}\text { Mean H days: } \\
\text { n }\end{array}$ & 12.02 & 11.37 & 12.16 \\
\hline In H deaths & $12(10.9)$ & $3(15.8)$ & $9(9.9)$ \\
\hline Mean NIHSS & \multicolumn{1}{|l|}{} \\
\hline Admission: $p$ & 8.62 & 12.84 & 7.73 \\
\hline Discharge: $p$ & 4.39 & 4.06 & 4.45 \\
\hline Vascular risk factors: $\mathbf{n}(\mathbf{\%})$ & $51(46.4)$ \\
\hline Hypertension & $58(52.7)$ & $7(6.4)$ & $51(20)$ \\
\hline $\begin{array}{l}\text { Diabetes } \\
\text { mellitus }\end{array}$ & $26(23.6)$ & $4(3.6)$ & $22(19.1)$ \\
\hline Hyperlipidemia & $28(25.4)$ & $7(6.4)$ & $21(19)$ \\
\hline $\begin{array}{l}\text { Atrial } \\
\text { fibrillation }\end{array}$ & $28(25.4)$ & $7(6.4)$ & $21(19.1)$ \\
\hline Stroke & $26(23.6)$ & $1(0.9)$ & $25(22.7)$ \\
\hline
\end{tabular}

NIHSS: NIH stroke scale, H days: hospitalization period, In $\mathrm{H}$ death: in hospital deaths. Values are number (\%) unless otherwise stated.

In our clinic, the mean hospitalization period of all patients in this study was 12.02 days. For the whole study group, the mean value of NIHSS was 8.62 points at admission and 4.39 points at discharge.

From a global perspective, during the hospitalization in our clinic, the degree of early recovery of neurological deficits was $49,07 \%$ (see fig. 1 ).

The percentage of patients who underwent thrombolytic therapy was $17.27 \%$. The mean value of NIHSS at admission and at discharge (A/D) were $12.84 / 4.06$ points for the patient with thrombolytic therapy and 7,73/4,45 points for the patients without thrombolytic therapy (see fig. 2).

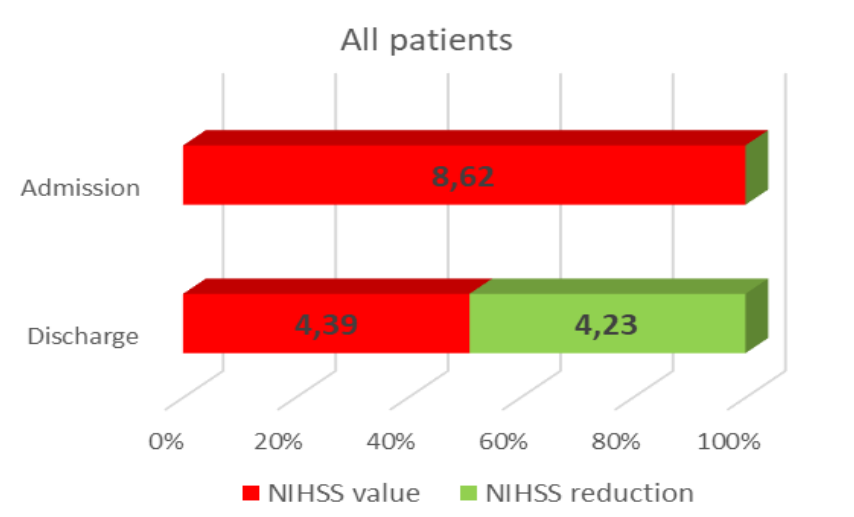

Fig. 1. Mean value of NIHSS for whole group of patients at admission/discharge and recovered NIHSS.
r-TPA treated/ non r-TPA treated patients

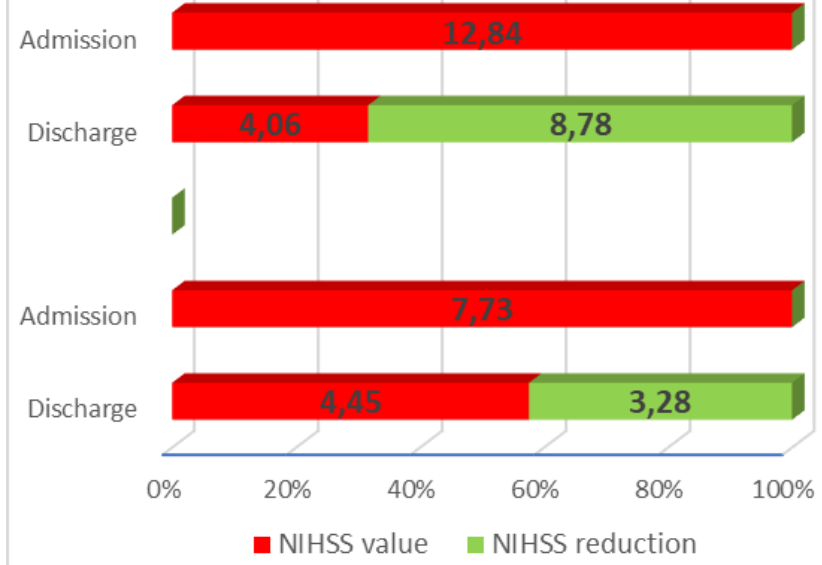

Fig. 2. Mean value of NIHSS for r-TPA treated (top) and non r-TPA treated(bottom)patients at admission/discharge and recovered NIHSS.

The mean value of in-hospital neurological recovery was superior for r-TPA treated patients (8.78 points in NIHSS score reduction) versus non r-TPA treated patients (3.28 points in NIHSS score reduction). The degree of early recovery of neurological deficits was $68,4 \%$ for first subgroup of patients and $42,4 \%$ for second subgroup. As expected, the highest grade of early recovery was observed in patients with thrombolytic therapy.

The data from our study revealed that facial and arm motor deficit with language functions were more affected (maximal scores), compared with cognitive and sensory functions (minimal scores). (see fig. 3)

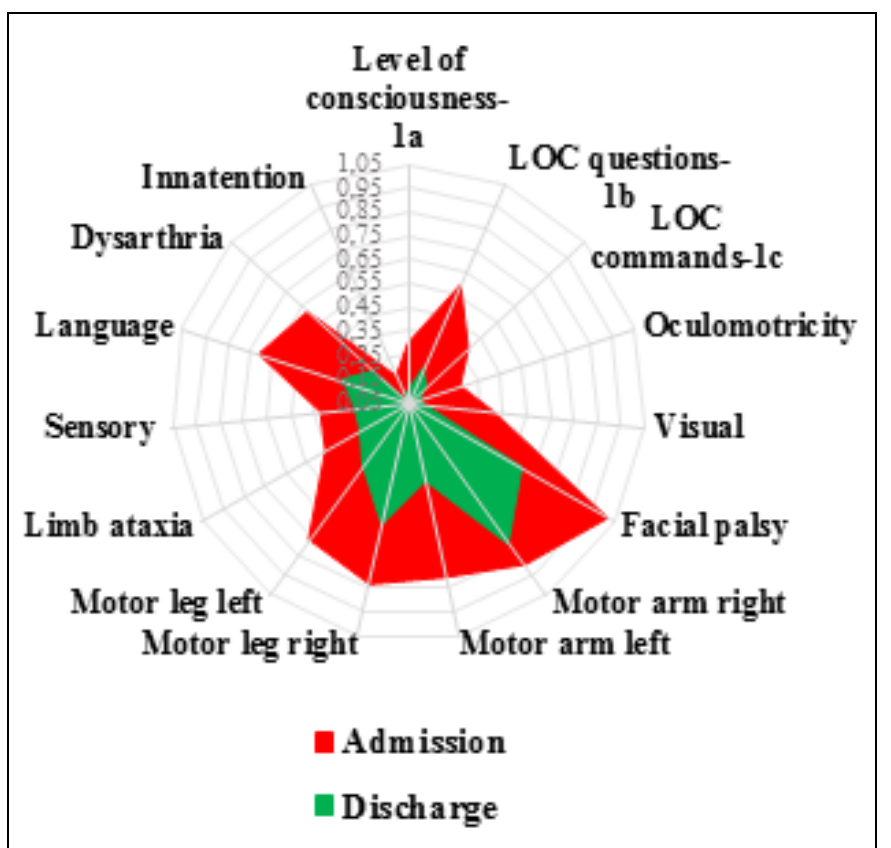

Fig. 3. Mean value of NIHSS items at admission and discharge for whole group of patients 
Studying the pattern of neurological damage in the first and in the last day of hospitalization in each patient from this study group, correlated with the access at r-TPA therapy, made us observe specific neurological patterns (see next radar type charts).

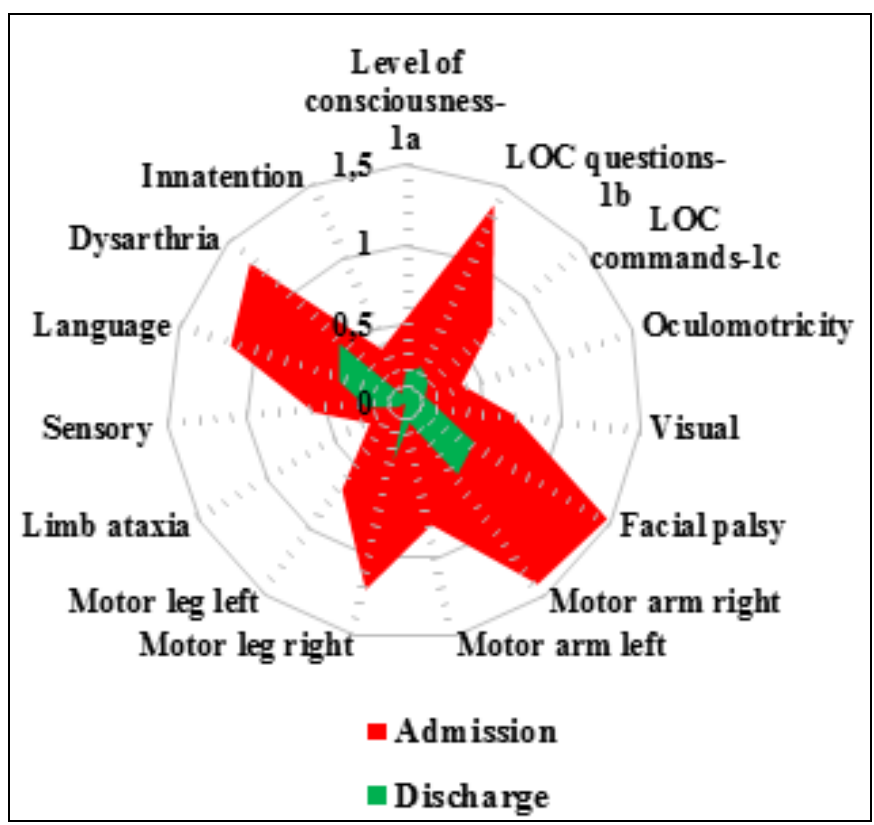

Fig. 4. Mean value of NIHSS items at admission and discharge for r-TPA treated patients

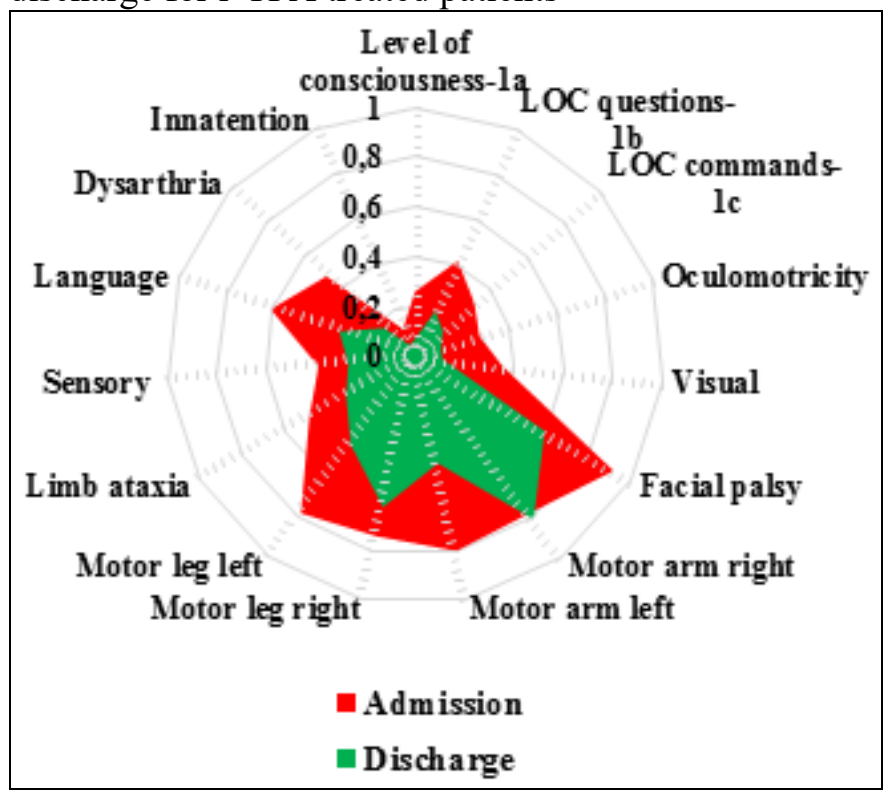

Fig. 5. Mean value of NIHSS items at admission and discharge for non r-TPA treated patients

Recovery of the motor deficit (lower/upper limbs and language) and verbal response was significantly greater in patients with thrombolytic therapy compared with the recovery of patients who did not received thrombolytic therapy. Recovery of sensitive and sensorial deficits has not varied significantly in the two therapeutic subgroups.

\section{Discussion}

Studies in humans and animal models show that most recovery from impairment occurs in the first 1-3 months after stroke as a result of both spontaneous recovery as well as increased responsiveness to enriched environments and training. Improvement from impairment is attributable to a short-lived "sensitive period" of post-stroke plasticity defined by unique genetic, physiological, and structural events (6).

Overall, trials of rehabilitation in the first 2 weeks after stroke are scarce. In the realm of very early mobilization, one large and one small trial found potential harm from mobilizing patients within the first $24 \mathrm{~h}$ after stroke, and only one small trial found benefit in doing so $(7,8)$.

However, thrombolytic treatment applied to patients who were admitted in the hospital in the first 4.5 hours window, significantly reduced the neurological deficit measured by the NIHSS from 12.84 to 4.06 points, averaging 8.78 points. Although the patients who underwent thrombolytic therapy had at the beginning a higher neurological damage than the other ones (12.84 vs. 7.73), at discharge their neurological status was better than that of those without thrombolytic therapy (4.06 vs. 4.45).

The NIHSS predicts post-acute care disposition among stroke patients. Predicting disposition on the first day of admission may facilitate the time-consuming and costly process of securing a bed at rehabilitation and perhaps decrease unnecessary length of stay in acute care settings $(4,8,9)$.

After the initial acute stroke therapy, applying the same rehabilitation methods in acute care settings, the hospitalization duration of patients who underwent thrombolysis was reduced by $8.33 \%$ compared to nonthrombolyzed (11.4 vs 12.2). Much more important was the extent of early recovery of neurological deficits $(68.4 \%$ vs. $42.4 \%)$. A high degree of early recovery from neurological deficits reduces the need for expensive care in the rehabilitation department $(7,10)$.

The degree of early recovery of non-thrombolyzed patients in this study (42.4\%) was comparable to the degree of early recovery (45.12\%) obtained in a similar study conducted in our clinic 5 years ago, when we did not have the option of thrombolytic therapy for patients with acute ischemic stroke. The severity at onset of ischemic stroke in thrombolyzed patients in the current study was comparable to that in patients in the previous study (mean NIHSS at admission of 12.84 points vs. 12.17 points) $(11,12,13)$. 


\section{Conclusions}

Early recovery of motor deficits is dependent on the type of therapy applied.

The degree of early recovery was superior for patients with thrombolytic therapy versus other conventional therapies ( $68,4 \%$ vs $42,4 \%)$.

The thrombolytic therapy reduced the average hospital care period (in acute care settings) with $8.33 \%$ for patients arriving at the hospital in a timely manner.

Continued efforts are needed to improve the response time of the population and emergency transport services to the hospital at the occurrence of an acute ischemic stroke, but also an increase in the number of emergency rooms and acute stroke units in which the application of thrombolytic therapy in optimal conditions is available.

\section{Declaration of conflict of interests/Conflict of Interest Statement}

The authors declare that there is no conflict of interest regarding the publication of this article.

\section{Informed consent}

Informed consent was obtained from all patients included in this study.

\section{References:}

1. Adams HP Jr., Davis PH, Leira EC, Chang KC, Bendixen BH, Clarke WR, et al. - Baseline NIH Stroke Scale Score Strongly Predicts Outcome atfer Stroke: A report of the Trial of Org 10172 in Acute Stroke Treatment (TOAST). Neurology. 1999;53(1):126-31. Available from: https://doi.org/10.1212/WNL.53.1.126

2. Lyden P. Using the National Institutes of Health Stroke Scale: a cautionary tale. Stroke. 2017; 48:513519. Available from: https://doi.org/10.1161/STROKEAHA.116.015434

3. Harrison JK, McArthur KS, Quinn TJ. Assessment scales in stroke: Clinimetric and clinical considerations. Clin Interv Aging. 2013;8:201-211. Available from: https://doi.org/10.2147/CIA.S32405

4. Muir KW, Weir CJ, Murray GD, Povey C, Lees KR. Comparison of neurological scales and scoring systems for acute stroke prognosis. Stroke. 1996;27(10);1817-1820. Available from:
https://doi.org/10.1161/01.STR.27.10.1817

5. Katzan IL, Thompson N, Uchino K. Innovations in stroke: the use of promis and neuroQol scales in clinical stroke trials. Stroke. 2016;47:e27-e30. Available https://doi.org/10.1161/STROKEAHA.115.011377

6. Zeiler SR. Should We Care About Early Post-Stroke Rehabilitation? Not Yet, but Soon. Curr Neurol Neurosci Rep. 2019;19(3):13. Available from: https://doi.org/10.1007/s11910-019-0927-x

7. Coleman ER, Moudgal R, Lang K. Early rehabilitation after stroke: a narrative review. Curr Atheroscler Rep.2017;19(12):59. Available from: https://doi.org/10.1007/s11883-017-0686-6

8. Yaghi S, Hinduja A, Bianchi N. Predictors of major improvement after intravenous thrombolysis in acute ischemic stroke. Int $\mathrm{J}$ Neurosci. 2016;126:67-69. Available from: https://doi.org/10.3109/00207454.2014.1002611

9. Schlegel D, Kolb SJ, Luciano JM, Tovar JM, Cucchiara BL, Liebeskind DS, et al. Utility of the NIH StrokeScale as a predictor of hospital disposition. Stroke. 2003;34:134-7. Available from: https://doi.org/10.1161/01.STR.0000048217.44714.02

10.Rondina JM, Park $\mathrm{CH}$, Ward NS. Brain regions important for recovery after severe post-stroke upper limb paresis. J Neurol Neurosurg Psychiatry. 2017; 88:737-743. Available from: https://dx.doi.org/10.1136/jnnp-2016-315030

11.Mutu CC, Spînu CȘ. The pattern of neurological damage in patients with ischemic stroke and their early recovery - study report. Acta Medica Transilvanica. 2019;24(3):63-65. Available from: http://www.amtsibiu.ro/Arhiva/2019/Nr3-en/Mutu.pdf

12.Kwah LK, Harvey LA, Diong J, Herbert RD. Models containing age and NIHSS predict recovery of ambulation and upper limb function six months after stroke: an observational study. Journal of physiotherapy. 2013;59(3);189-197. Available from: https://doi.org/10.1016/S1836-9553(13)70183-8

13.Lee KB, Lim SH, Kim KH, Kim KJ, Kim YR, Chang $\mathrm{WN}$, et al. Six-month functional recovery of stroke patients: a multi-time-point study. Int J Rehabil Res. 2015;38(2):173-180. Available from: https://pubmed.ncbi.nlm.nih.gov/25603539 